\title{
Structure of the membrane-intrinsic nitric oxide reductase from Roseobacter denitrificans
}

\author{
Allister Crow ${ }^{1 *}$, Yuji Matsuda ${ }^{2}$, Hiroyuki Arata $^{2}$, Arthur Oubrie $^{3 *}$ \\ ${ }^{1}$ Department of Pathology, University of Cambridge, UK. 'Department of Biology, Kyushu University, \\ Fukuoka, Japan. ${ }^{3}$ Lead Pharma, Pivot Park, 5349AC Oss, The Netherlands.
}

*Corresponding authors: ac800@cam.ac.uk \& arthur.oubrie@leadpharma.com

\begin{abstract}
Membrane-intrinsic nitric oxide reductases (NORs) are key components of bacterial denitrification pathways with a close evolutionary relationship to the cytochrome oxidase (COX) complex found in aerobic respiratory chains. A key distinction between COX and NOR is the identity of the metal directly opposite the heme $b_{3}$ within the active site. In NOR, this metal is iron $\left(\mathrm{Fe}_{\mathrm{B}}\right)$ whereas in $\mathrm{COX}$ it is copper $\left(\mathrm{Cu}_{\mathrm{B}}\right)$. The purified NOR of Roseobacter denitrificans contains copper and has modest oxidase activity raising the possibility that a COX-like active site might have independently arisen within the context of a NOR-like protein scaffold. Here we present the crystal structure of the Roseobacter denitrificans NorBC complex and anomalous scattering experiments probing the identity of each metal centre. Our results refute the hypothesis that copper occupies the active site and instead reveal a new metal centre in the small subunit not seen in any other NOR or COX.
\end{abstract}

\section{INTRODUCTION}

Membrane-bound nitric oxide reductases catalyse the conversion of nitric oxide (NO) to nitrous oxide $\left(\mathrm{N}_{2} \mathrm{O}\right)$, a key step in the process of denitrification ${ }^{1}$. Bacterial denitrification has significant impact on the global climate through the production of $\mathrm{NO}$ and $\mathrm{N}_{2} \mathrm{O}$ which are both more potent greenhouse gases than $\mathrm{CO}_{2}{ }^{2}$. Nitric oxide reductase activity is also used by bacteria to evade destruction by macrophages and other immune cells that produce nitric oxide to kill invading pathogens ${ }^{3}$. To date, two membrane-intrinsic bacterial NOR proteins have been structurally characterised ${ }^{3-5}$. The Pseudomonas aeruginosa $\mathrm{NOR}^{3}$, hereon termed PaNOR, is typical of the 'cNOR' class of enzymes that possess a c-type cytochrome component and accept electrons from periplasmic and membranebound electron donors such as $c$-type cytochromes and pseudoazurin ${ }^{6}$. The $P$. aeruginosa enzyme was crystallised with the aid of an antibody raised against the small subunit, and was purified as a two-component enzyme composed of a large (NorB) and small subunit (NorC) ${ }^{3}$. In contrast, the enzyme from Geobacillus denitrificans ${ }^{5}$, GsNOR, is typical of the 'qNOR' class, comprises a single polypeptide (NorZ) and accepts electrons from the quinol pool. A third NOR class is the ' $\mathrm{Cu}_{\mathrm{A}} \mathrm{NOR}$ ' from Bacillus azotoformans, a heterotrimeric NOR that appears to contain a small subunit di-copper site and accepts electrons from $c$-type cytochromes ${ }^{7}$. No structures are yet available for the $\mathrm{Cu}_{A} \mathrm{NOR}$ class. The large subunits of each of these metalloproteins share significant sequence similarity to the cytochrome/quinol oxidase family of proteins $^{8}$ for which multiple enzymes have been structurally characterised $^{9-12}$. Cytochrome/quinol oxidases are fundamental components of aerobic respiratory 
chains that facilitate use of molecular oxygen as a terminal electron acceptor. The nitric oxide reductase (NOR) and cytochrome/quinol oxidase (COX) families share a common ancestor, and together form a class of membrane protein termed the Heme:copper oxidase (HCO) superfamily.

A key feature distinguishing $\mathrm{COX}$ from NOR is the presence of a copper ion $\left(\mathrm{Cu}_{B}\right)$ within the active site in place of the iron $\left(\mathrm{Fe}_{\mathrm{B}}\right)$ found at an equivalent position in NORs. The NorBC protein of $R$. denitrificans shares a high degree of sequence similarity with other characterised NOR enzymes, but has been shown to contain copper and possess a weak oxidase activity raising the intriguing possibility that a copper ion may have been incorporated into a NOR-like active site ${ }^{1,13,14}$. To further investigate this unique metalloenzyme, we determined the crystal structure of the $R$. denitrificans NorBC complex (hereon termed RdNOR) and scrutinized the identity of the active site metal ions using anomalous $\mathrm{X}$-ray scattering experiments.

\section{EXPERIMENTAL PROCEDURES}

\section{Protein production and crystallisation}

Preparation of the RdNorBC complex has been described previously ${ }^{13}$. Protein prepared for this work was generated in the same manner, except that, after purification in sucrose monocaprate, RdNorBC was exchanged into nonyl- $\beta$-D-glucopyranoside using cycles of concentration and dilution in a highpressure stirred filter device (Amicon). For crystallization, the RdNorBC complex was first exchanged into a buffer composed of $20 \mathrm{mM}$ MOPS pH 7.2, $100 \mathrm{mM} \mathrm{NaCl}, 0.4 \%$ (w/v) nonyl- $\beta-\mathrm{D}-$ glucopyranoside and concentrated to $\sim 8 \mathrm{mg} / \mathrm{ml}$. Crystals were obtained using the sitting drop crystallization method at $4{ }^{\circ} \mathrm{C}$ using a crystallization reagent composed of $100 \mathrm{mM}$ MOPS pH 7.6, $28 \%$ (w/v) PEG 600. The crystallization drop was formed by mixing the protein solution and crystallization reagent in a 1:1 ratio over an $800 \mu \mathrm{l}$ volume of the crystallization reagent alone. Crystals typically appeared overnight, but larger crystals grew over several days. Crystals were frozen in a cryoprotectant formed by diluting the mother liquor with ethylene glycol to $20 \%(\mathrm{v} / \mathrm{v})$.

\section{Crystal structure determination}

X-ray diffraction data were collected at the European Synchrotron Radiation Facility. Structure determination and analysis used programs from the $C C P 4^{15}$ and $\mathrm{RAVE}^{16}$ software suites. X-ray diffraction data were indexed and integrated with IMOSFLM ${ }^{17}$ and then scaled with SCALA ${ }^{18}$. Phases were obtained by the molecular replacement method as implemented in PHASER ${ }^{19}$ using a severelypruned polyalanine model derived from the structure of Pseudomonas aeruginosa NOR (pdb entry $300 \mathrm{R}^{3}$ ) as a molecular replacement probe. Phases were further improved by density modification using PARROT ${ }^{20}$, before the model was re-built using BUCCANEER ${ }^{21}$. The amino acid sequence of NorB and NorC were deduced from the genomic DNA sequence of Roseobacter denitrificans ${ }^{22}$. The structure was completed using iterative cycles of manual model-building and refinement using $\mathrm{COOT}^{23}$ and REFMAC ${ }^{24}$, respectively. As refinement neared completion, validation tools from $\mathrm{COOT}^{23}$ and MOLPROBITY ${ }^{25}$ were employed to maximise the quality of the model. Metal site validation was 
assisted by the Check My Metal server ${ }^{26}$. Structural superpositions used CCP4 SUPERPOSE with the secondary structure matching algorithm ${ }^{27}$ and interface analysis used PISA ${ }^{28}$.

\section{Anomalous difference map analysis}

Anomalous scattering maps were prepared using CAD and FFT as implemented in CCP4 $4^{15}$. Anomalous differences were derived from each of the two datasets during integration and the maps were calculated using phases derived from the refined model. Integration of the anomalous scattering at each metal site was conducted using the PEEK SPHERE function of MAPMAN ${ }^{16}$. Anomalous scattering maps were integrated using a $2.05 \AA$ radius of integration to avoid problems of overlapping the integration ranges of the active site ions. Anomalous scattering map integrals were placed on a common scale with f" values expected from theory using a simple scale factor to place the mean scattering values of the three heme sites at the expected theoretical f" value for each wavelength.

\section{Active site ligand identification}

Difference electron density maps $\left(|F|_{o b s}{ }^{-}|F|_{c a l c}\right)$ were calculated for multiple different models of the active site (unoccupied, $\mu$-oxo bridged and dioxygen bound, peroxo bridged and chloride bound) using FFT after refinement with REFMAC. In the deposited coordinates, the ligand was refined as dioxygen but marked UNK to reflect remaining ambiguity in the identity of the diatomic bridging ligand.

\section{RESULTS}

RdNOR was purified directly from Roseobacter denitriifcans membranes using nonyl- $\beta$-Dglucopyranoside as the final stabilising detergent and crystallised using small molecular weight PEGs at $\mathrm{pH} 7.6$ (see methods). Crystals of RdNOR belong to space group P22 ${ }_{1}{ }_{2}$, contain a single NorBC complex in the asymmetric unit and have a $69 \%$ solvent content. X-ray data extends to $2.8 \AA$ resolution allowing a complete atomic model to be built and refined with an $R_{\text {work }}$ and $R_{\text {free }}$ of 0.198 and 0.247. Statistics for the X-ray datasets and refined atomic model are given in Table $\mathbf{S} 1$ and the overall structure is shown in Figure 1. A movie summarizing the structural features of RdNOR and the location of the membrane-spanning region is also available (Supplemental Movie 1).

RdNOR belongs to the cNOR class of nitric oxide reductases and can be superposed with PaNOR with an rmsd of $0.84 \AA$ (for 555 matched Ca positions). RdNOR was purified as a complex of two subunits (NorB and NorC) that are tightly associated (Fig. 1a). The total buried surface area of the NorBC interface is $3,156 \AA^{2}$ corresponding to $16 \%$ of the surface of NorB and $33 \%$ of NorC. The small subunit, NorC, is a $16 \mathrm{kDa}$ protein containing a single heme that is covalently bound within a classical CXXCH motif (specifically Cys62-Val63-Asn64-Cys65-His66 in this case) and possesses a single transmembrane helix at its $\mathrm{N}$-terminus that packs closely with the transmembrane helices of the larger subunit. The $c$-type heme iron is coordinated by the side chains of Met116 and His66 (Fig. 1b). The large subunit (51 kDa NorB) has 12 transmembrane helices and encompasses a $b$-type heme (bis-ligated by His53 and His336, Fig. 1c) and the active site (Fig. 1d) which is composed of a single non-covalently bound heme (termed heme $b_{3}$ ) ligated by His334 and another transition-element ion 
that is bound in close proximity to the heme iron. This metal ion is ligated to NorB residues His194, Glu198, His245 and His246. The identity of this metal is of central interest to this study, because it has previously been suggested that the Roseobacter enzyme contains copper and that this may reside in the active site in place of the iron $\left(\mathrm{Fe}_{\mathrm{B}}\right)$ that is typically found in $\mathrm{NORs}^{13,14}$. The two hemes found in NorB are bridged by a single calcium ion with interactions arising from both heme propionyl groups as well as atoms derived from both the large and small subunits of the enzyme (Fig. 1e). In addition to these conventional sites, a further metal ion is found in the NorC subunit, bound at the periplasmfacing surface by residues His52, Glu49 and Glu124 (Fig. 1f). The central ion adopts tetrahedral coordination geometry with a crystallographically-ordered water molecule as the fourth ligand. Both glutamates coordinate in monodentate fashion with coordination by His52 occurring via the $\delta$-nitrogen atom. This site has not been observed in any other nitric oxide reductase or cytochrome oxidase complex.

To determine the identity of the metal sites adjacent to heme $b_{3}$ (i.e. within the active site) and within the small subunit, we performed X-ray anomalous scattering experiments. X-ray datasets were collected at two wavelengths $(0.976 \AA$ and $1.739 \AA)$ and the magnitude of the anomalous scattering quantified at each metal centre using local integration of the anomalous difference map (Figure 2). Integrated values from the anomalous scattering map and theoretical f" values for each metal site are summarized in Table S2. Since the identity of the metals at the centre of each heme moiety is not in doubt, we used these sites as internal reference values for comparison with other sites. The anomalous scattering from the site opposite heme $b_{3}$ has similar magnitude to that of the three heme irons for both wavelengths (Figs. 2a,b) suggesting it is iron. On the other hand, the small subunit metal ion scatters strongly at $0.976 \AA$ (Fig. 2b) but not at $1.739 \AA$ (Fig. 2a) consistent with either copper or zinc, but not with iron (Fig. 2c). The anomalous scattering experiments thus confirm that RdNOR has a conventional NOR-like di-iron active site similar to that seen previously in the structure of $\mathrm{PaNOR}^{3}$ rather than a mixed iron:copper site that is characteristic of COX proteins. The identity of the small subunit metal is consistent with copper (explaining previous biochemical detection) but the possibility of $\mathrm{Zn}$ cannot be excluded by the anomalous scattering data alone.

Importantly, we note that the small subunit metal site located on the NorC protein surface appears to be unique to the Roseobacter enzyme, its location and structure being distinct from the $\mathrm{Cu}_{\mathrm{A}}$ binuclear centre found in cytochrome $b a_{3}$ oxidase $^{12}, c a a_{3}$ oxidase $^{11}, a_{3}$ oxidase $^{9}$ and that proposed for $\mathrm{Cu}_{\mathrm{A}} \mathrm{NOR}$ of Bacillus azotoformans ${ }^{7}$, and so we do not suspect any evolutionary link with any of these sites.

It is unclear what role, if any, the newly discovered small subunit metal plays in RdNOR. Electron transfer between this site and the $c$-heme is feasible, but the distance separating these potential redox centres is relatively large (14 $\AA$ edge-to-edge or $19.7 \AA$ centre-to-centre) suggesting the kinetics of such transfers would be slow ${ }^{29}$. Furthermore, metal-coordinating amino acids are not conserved among other NORs (see alignment in Fig. S1) suggesting the site may be dispensible or else serves a 
specific function only in $R$. denitrificans. Alternative roles for the small subunit metal site that do not involve electron transfer may be to mediate interactions with native electron donors such as cytochrome $c$ or pseudoazurin, confer protein stability, or else to bind further NOR subunits or other respiratory complexes.

The active site of RdNOR shares many similarities with PaNOR including conservation of all $\mathrm{Fe}_{B}$ coordinating residues in the active site and an equivalent network of tunnels and cavities that allow ingress and egress of substrates and products. However, the distance between the iron of heme $b_{3}$ and the adjacent $\mathrm{Fe}_{\mathrm{B}}$ is longer in RdNOR $(4.3 \AA)$ than in the as-purified structure of PaNOR (3.8 $\AA$ ). The inter-iron distance in RdNOR is much closer to that observed in the structures of reduced or CObound $\mathrm{PaNOR}^{30}$ (the latter of which has been proposed to mimic the NO-bound form of the enzyme) although no reducing agents or diatomic ligands such as $\mathrm{CO}$ or NO were added in preparation of RdNOR crystals. In addition, while as-purified PaNOR has bridging electron density between the two irons consistent with a $\mu$-oxo bridge, the active site of RdNOR contains additional electron density suggesting a higher molecular weight ligand.

To characterise the RdNOR ligand, we built and refined multiple models for the ligand-bound active site and used the difference electron density maps to assess which of these provide good fits to the data, including a control where no bridging ligand was included. Models lacking a ligand (Fig. S2a) or else containing a $\mu$-oxo bridge or single water (Fig. S2b) do not adequately describe the data as judged by the presence of significant difference electron density. Models incorporating diatomic molecules such as dioxygen do give plausible fits (Fig. S2c), but at $2.8 \AA$ resolution it is not possible to accurately define their orientation or to discern between different atom types (e.g. carbon, oxygen or nitrogen). We conclude that the active site is probably occupied by dioxygen or dinitrogen, while recognising that a peroxo-bridge similar to that suggested for cytochrome oxidase ${ }^{31}$ might also fit the available data.

\section{DISCUSSION}

RdNOR is the third nitric oxide reductase for which a crystal structure has been resolved. Structures of PaNOR and GsNOR have considerably different active site configurations. PaNOR contains a di-iron site composed of one heme and one iron atom ligated to three histidines and a glutamate ${ }^{4}$. In contrast, the active site of GsNOR contains a $\mathrm{Zn}$ (II) ion directly opposite the heme iron, with a pair of water molecules displacing the equivalent glutamate ${ }^{5}$. The PaNOR active site configuration matches the most commonly proposed NOR active site model based on spectroscopic data, but the active site of GsNOR does not. Consistency between the active site of RdNOR and PaNOR suggests a classical diiron site configuration is the norm and that the presence of Zinc in GsNOR is artefactual. The diversity of active site structures recorded for crystallographically-resolved NORs is interesting, but it remains difficult to assess the physiological relevance of each due to the inevitable difficulty in interpreting active site density at the modest resolutions typical of membrane protein structures. Further higherresolution structures obtained under carefully controlled redox conditions directly coupled with detailed 
spectroscopic analysis (both in solution and in crystallo) are essential to fully understand and catalogue the NOR catalytic mechanism in molecular detail.

The nitric oxide reductase of $R$. denitrificans has previously been shown to contain copper and possess oxidase activity leading to the suggestion that the active site iron may have been substituted by copper to form a COX-like enzymatic centre within a NOR protein scaffold. Evidence for the presence of copper in the active site of RdNOR was based primarily on spectroscopic and biochemical techniques which revealed an EPR-silent anti-ferromagnetically coupled active site ${ }^{14}$ and ICP-MS metal analysis showing the presence of one tightly-bound copper ion per NorBC complex ${ }^{13}$. Subsequent work by other groups whereby the genes encoding RdNOR were deleted have shown that nitric oxide reductase activity of $R$. denitrifcans is dependent on NorBC, strongly suggesting it is a true nitric oxide reductase ${ }^{32}$, but the presence of copper was not accounted for. The structural data presented here clarifies the situation considerably, demonstrating that RdNOR contains a typical NOR-like heme-Fe: $\mathrm{Fe}_{\mathrm{B}}$ active site with a probable copper site located in the small subunit. Under this revised model for RdNOR structure, the observed di-iron site is still expected to be EPR-silent, as is the case for other purified NORs, and the crystallographically observed metal content is set in better agreement with that determined biochemically ${ }^{13}$ than for the previous model in which copper was proposed to occupy the active site.

In summary, we have presented the $2.8 \AA$ crystal structure of the membrane-intrinsic NorBC complex from $R$. denitrificans and show that, counter to previous orthodoxy, the protein contains a di-iron active site and a previously unanticipated metal within the small subunit. The addition of the small subunit copper/zinc to nitric oxide reductase appears to have occurred relatively recently from an evolutionary perspective and perhaps only within $R$. denitrificans. The discovery of the small subunit metal site in RdNOR further underlines the structural diversity found within the heme copper oxidase superfamily.

\section{ACKNOWLEDGMENTS}

We thank staff at the ESRF for synchrotron facilities.

\section{SUPPORTING INFORMATION}

Supplementary figures and tables are available as a PDF and a supplemental movie showing key features of the RdNorBC structure is available in '.mpg' format. Coordinates are deposited with the Protein Data Bank, accession code 4XYD. Diffraction images are available from the Zenodo archive DOI10.5281/zenodo.23388.

\section{AUTHOR CONTRIBUTIONS}

YM grew Roseobacter and purified protein under supervision of $\mathrm{HA} ; \mathrm{AC}$ screened detergents and crystallised RdNorBC under supervision of $A O ; A C$ solved the structure, conducted the anomalous scattering analysis and wrote the paper; $A C$ and $A O$ finalised the manuscript. 


\section{ABBREVIATIONS}

NOR - Nitric Oxide Reductase

COX - Cytochrome $c$ Oxidase

NO - Nitric oxide

ICP-MS - Inductively coupled plasma - mass spectrometry

EPR - Electron Paramagnetic Resonance

\section{FIGURE LEGENDS}

Figure 1. Key structural features of Roseobacter denitrificans NorBC. (a) Overall structure of the RdNorBC complex determined at $2.8 \AA$ resolution. (b) Covalently-bound heme $c$ in the small subunit. (c) Heme $b$ in the large subunit. (d) The active site containing an unidentified diatomic ligand. (e) Heme-bridging calcium. (f) The unique small subunit metal site. Throughout the panel, the large subunit (NorB) is shown in green and small subunit (NorC) shown in blue. Metal ions are shown as solid spheres; iron is orange, Calcium is magenta, and the small subunit copper/zinc is yellow.

Figure 2. Anomalous scattering experiments used to assign elemental identities to each metal site. (a) Data collected at $7,130 \mathrm{eV}$. (b) Data collected at $12,700 \mathrm{eV}$. Anomalous scattering maps are contoured at $3.75 \sigma$. Values indicate the integrated anomalous scattering density at each metal centre in units proportional to electrons. (c) Theoretical curves showing the expected magnitude of the anomalous scattering component $\left(f^{\prime \prime}\right)$ for copper, iron and calcium.

\section{REFERENCES}

(1) Zumft, W. G. (2005) Nitric oxide reductases of prokaryotes with emphasis on the respiratory, heme-copper oxidase type. J. Inorg. Biochem. 99, 194-215

(2) Canfield, D. E., Glazer, A. N., and Falkowski, P. G. (2010) The evolution and future of Earth's nitrogen cycle. Science 330, 192-196.

(3) Hino, T., Matsumoto, Y., Nagano, S., Sugimoto, H., Fukumori, Y., Murata, T., Iwata, S., and Shiro, Y. (2010) Structural basis of biological $\mathrm{N}_{2} \mathrm{O}$ generation by bacterial nitric oxide reductase. Science $330,1666-1670$.

(4) Hino, T., Nagano, S., Sugimoto, H., Tosha, T., and Shiro, Y. (2012) Molecular structure and function of bacterial nitric oxide reductase. Biochim. Biophys. Acta 1817, 680-7.

(5) Matsumoto, Y., Tosha, T., Pisliakov, A. V, Hino, T., Sugimoto, H., Nagano, S., Sugita, Y., and Shiro, Y. (2012) Crystal structure of quinol-dependent nitric oxide reductase from Geobacillus stearothermophilus. Nat. Struct. Mol. Biol. 19, 238-245

(6) Thorndycroft, F. H., Butland, G., Richardson, D. J., and Watmough, N. J. (2007) A new assay for nitric oxide reductase reveals two conserved glutamate residues form the entrance to a protonconducting channel in the bacterial enzyme. Biochem. J. 401, 111-119. 
(7) Al-Attar, S., and de Vries, S. (2015) An electrogenic nitric oxide reductase. FEBS Lett. 589, 2050 2057.

(8) Hendriks, J., Oubrie, A., Castresana, J., Urbani, A., Gemeinhardt, S., and Saraste, M. (2000) Nitric oxide reductases in bacteria. Biochim. Biophys. Acta - Bioenerg. 1459, 266-273.

(9) Iwata, S., Ostermeier, C., Ludwig, B., and Michel, H. (1995) Structure at 2.8 A resolution of cytochrome c oxidase from Paracoccus denitrificans. Nature. 376, 660-669.

(10) Lyons, J. A., Aragão, D., Slattery, O., Pisliakov, A. V., Soulimane, T., and Caffrey, M. (2012) Structural insights into electron transfer in caa $_{3}$-type cytochrome oxidase. Nature. 487, 514-518

(11) Buschmann, S., Warkentin, E., Xie, H., Langer, J. D., Ermler, U., and Michel, H. (2010) The structure of $c b b_{3}$ cytochrome oxidase provides insights into proton pumping. Science 329, 327-330.

(12) Soulimane, T., Buse, G., Bourenkov, G. P., Bartunik, H. D., Huber, R., and Than, M. E. (2000) Structure and mechanism of the aberrant $b a(3)$-cytochrome $c$ oxidase from thermus thermophilus. EMBO J. 19, 1766-1776.

(13) Matsuda, Y., Inamori, K., Osaki, T., Eguchi, A., Watanabe, A., Kawabata, S., Iba, K., and Arata, $\mathrm{H}$. (2002) Nitric oxide-reductase homologue that contains a copper atom and has cytochrome $c$ oxidase activity from an aerobic phototrophic bacterium Roseobacter denitrificans. J. Biochem. 131, 791-800.

(14) Matsuda, Y., Uchida, T., Hori, H., Kitagawa, T., and Arata, H. (2004) Structural characterization of a binuclear center of a Cu-containing NO reductase homologue from Roseobacter denitrificans: EPR and resonance Raman studies. Biochim. Biophys. Acta - Bioenerg. 1656, 37-45.

(15) Winn, M. D., Ballard, C. C., Cowtan, K. D., Dodson, E. J., Emsley, P., Evans, P. R., Keegan, R. M., Krissinel, E. B., Leslie, A. G. W., McCoy, A., McNicholas, S. J., Murshudov, G. N., Pannu, N. S., Potterton, E. A., Powell, H. R., Read, R. J., Vagin, A., and Wilson, K. S. (2011) Overview of the CCP4 suite and current developments. Acta Crystallogr. Sect. D Biol. Crystallogr. 67, 235-242

(16) Kleywegt, G. J., and Jones, T. A. (1996) xdIMAPMAN and xdIDATAMAN - Programs for reformatting, analysis and manipulation of biomacromolecular electron-density maps and reflection data sets. Acta Crystallogr. Sect. D Biol. Crystallogr. 52, 826-828.

(17) Battye, T. G. G., Kontogiannis, L., Johnson, O., Powell, H. R., and Leslie, A. G. W. (2011) iMOSFLM: A new graphical interface for diffraction-image processing with MOSFLM. Acta Crystallogr. Sect. D Biol. Crystallogr. 67, 271-281.

(18) Evans, P. (2006) Scaling and assessment of data quality, in Acta Crystallographica Section D: Biological Crystallography, 62, 72-82.

(19) McCoy, A. J., Grosse-Kunstleve, R. W., Adams, P. D., Winn, M. D., Storoni, L. C., and Read, R. J. (2007) Phaser crystallographic software. J. Appl. Crystallogr. 40, 658-674.

(20) Cowtan, K. (2010) Recent developments in classical density modification. Acta Crystallogr. Sect. D Biol. Crystallogr. 66, 470-478.

(21) Cowtan, K. (2006) The Buccaneer software for automated model building. 1. Tracing protein chains. Acta Crystallogr. Sect. D Biol. Crystallogr. 62, 1002-1011.

(22) Swingley, W. D., Sadekar, S., Mastrian, S. D., Matthies, H. J., Hao, J., Ramos, H., Acharya, C. R., Conrad, A. L., Taylor, H. L., Dejesa, L. C., Shah, M. K., O'Huallachain, M. E., Lince, M. T., 
Blankenship, R. E., Beatty, J. T., and Touchman, J. W. (2007) The complete genome sequence of Roseobacter denitrificans reveals a mixotrophic rather than photosynthetic metabolism. J. Bacteriol. 189, 683-690.

(23) Emsley, P., Lohkamp, B., Scott, W. G., and Cowtan, K. (2010) Features and development of Coot. Acta Crystallogr. Sect. D Biol. Crystallogr. 66, 486-501.

(24) Murshudov, G. N., Skubák, P., Lebedev, A. A., Pannu, N. S., Steiner, R. A., Nicholls, R. A., Winn, M. D., Long, F., and Vagin, A. A. (2011) REFMAC5 for the refinement of macromolecular crystal structures. Acta Crystallogr. Sect. D Biol. Crystallogr. 67, 355-367.

(25) Chen, V. B., Arendall, W. B., Headd, J. J., Keedy, D. A., Immormino, R. M., Kapral, G. J., Murray, L. W., Richardson, J. S., and Richardson, D. C. (2010) MolProbity: All-atom structure validation for macromolecular crystallography. Acta Crystallogr. Sect. D Biol. Crystallogr. 66, 12-21.

(26) Zheng, H., Chordia, M. D., Cooper, D. R., Chruszcz, M., Müller, P., Sheldrick, G. M., and Minor, W. (2014) Validation of metal-binding sites in macromolecular structures with the CheckMyMetal web server. Nat. Protoc. 9, 156-70.

(27) Krissinel, E., and Henrick, K. (2004) Secondary-structure matching (SSM), a new tool for fast protein structure alignment in three dimensions. Acta Crystallogr. Sect. D Biol. Crystallogr. 60, 22562268.

(28) Krissinel, E., and Henrick, K. (2007) Inference of macromolecular assemblies from crystalline state. J. Mol. Biol. 372, 774-97.

(29) Gray, H. B., and Winkler, J. R. (2005) Long-range electron transfer. Proc. Natl. Acad. Sci. U. S. A. 102, 3534-3539.

(30) Sato, N., Ishii, S., Sugimoto, H., Hino, T., Fukumori, Y., Sako, Y., Shiro, Y., and Tosha, T. (2014) Structures of reduced and ligand-bound nitric oxide reductase provide insights into functional differences in respiratory enzymes. Proteins Struct. Funct. Bioinforma. 82, 1258-1271.

(31) Koepke, J., Olkhova, E., Angerer, H., Müller, H., Peng, G., and Michel, H. (2009) High resolution crystal structure of Paracoccus denitrificans cytochrome $c$ oxidase: new insights into the active site and the proton transfer pathways. Biochim. Biophys. Acta 1787, 635-645.

(32) Kimura, M., Ishii, M., Igarashi, Y., and Arai, H. (2012) Identification of the Genes Encoding Nitric Oxide Reductase in the Aerobic Photosynthetic Bacterium Roseobacter denitrificans OCh114. Biosci. Biotechnol. Biochem. 76, 1984-1986. 
Figure 1.

a
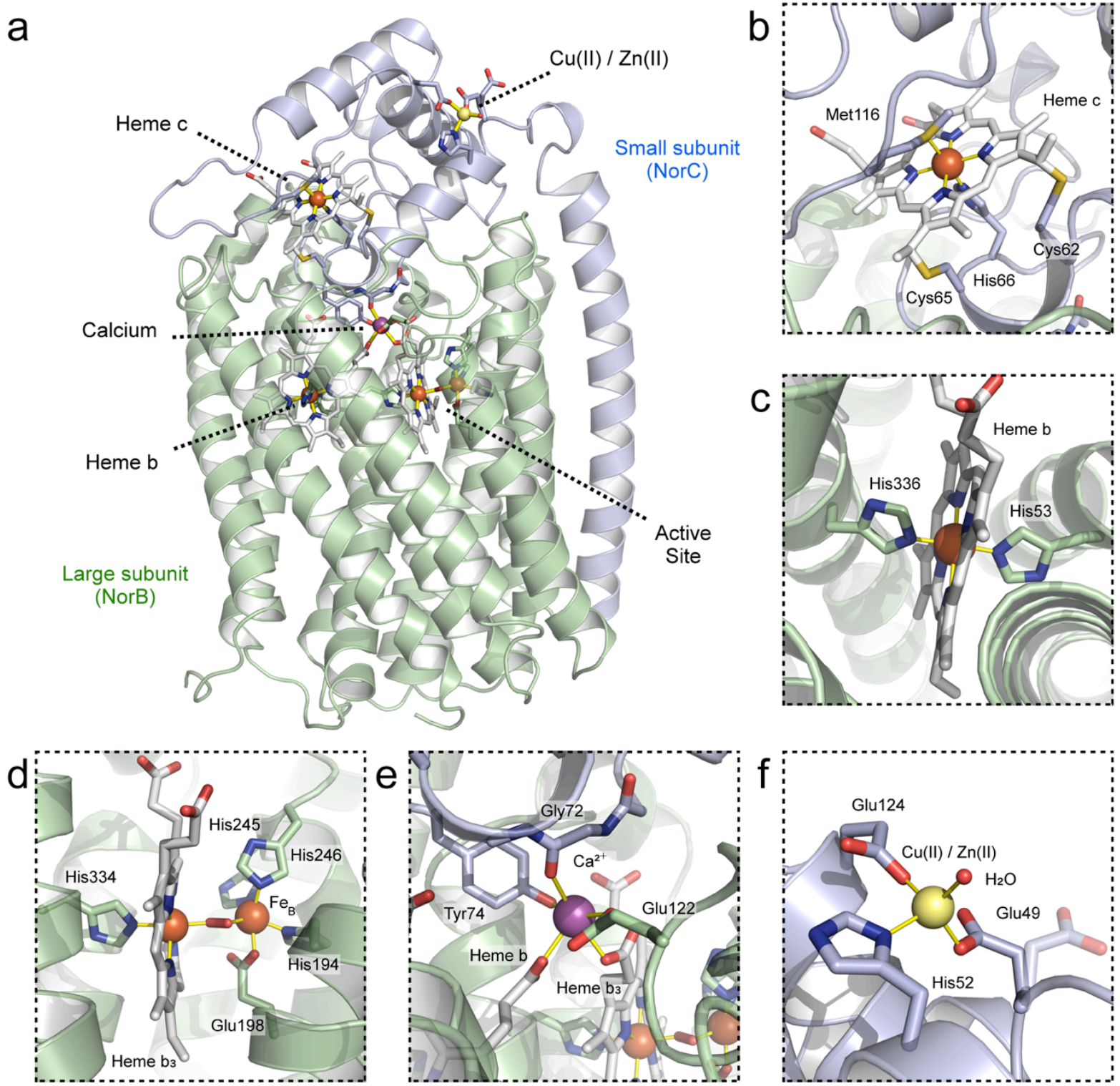


\section{Figure 2}

a
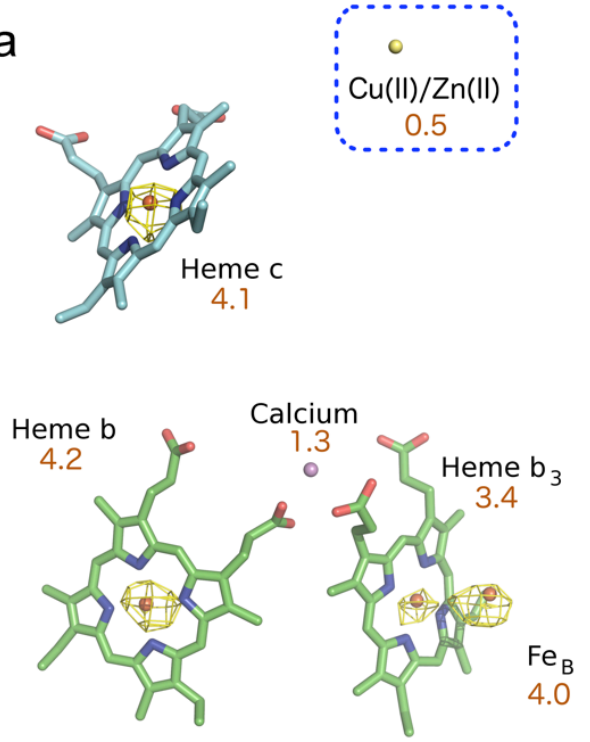

b
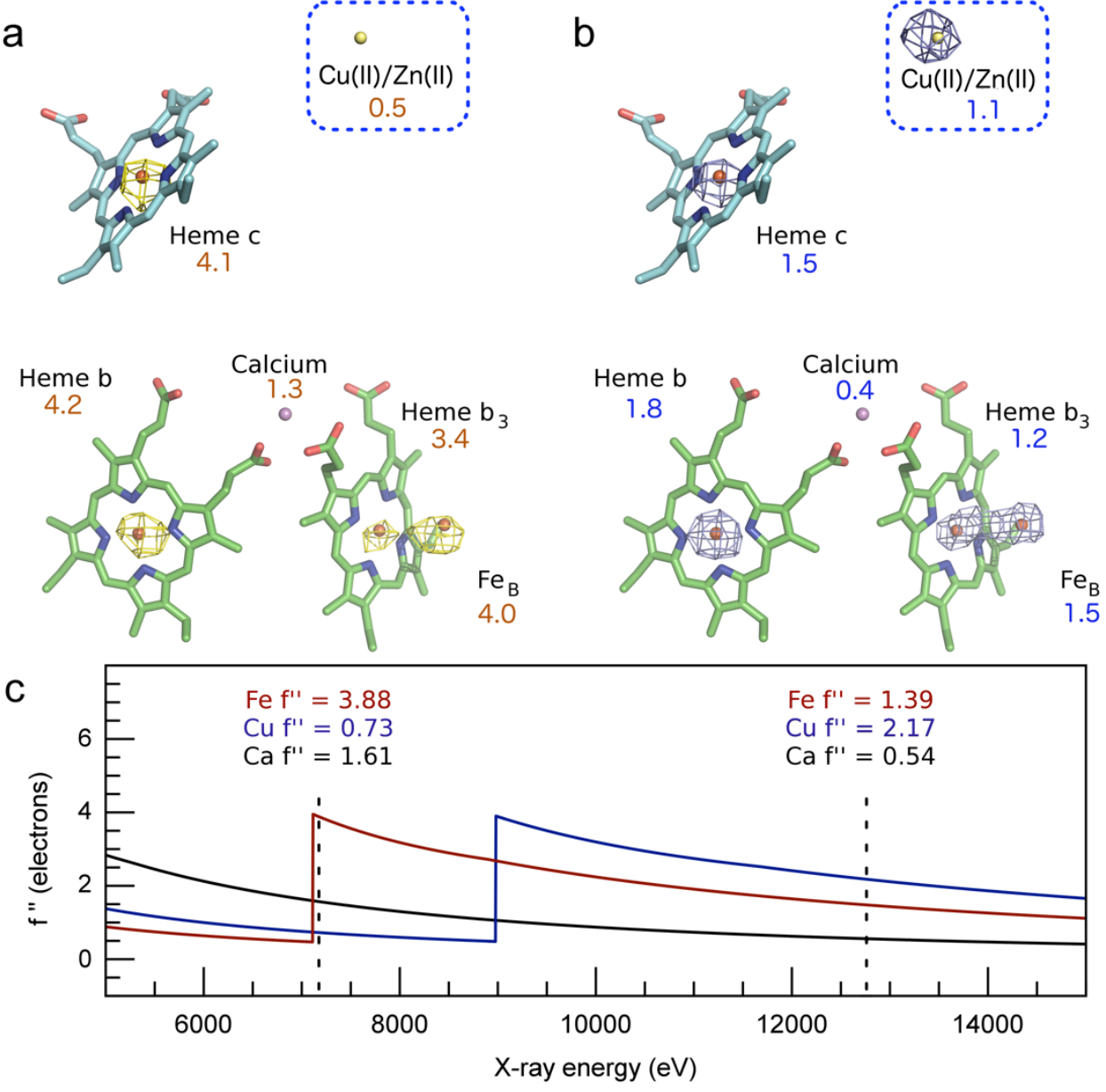
FOR TABLE OF CONTENTS ONLY

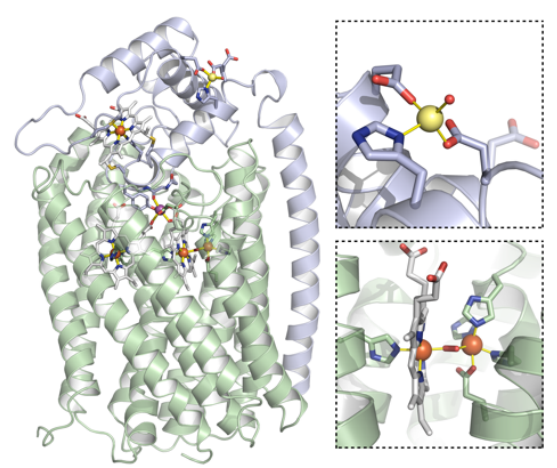




\section{SUPPLEMENTARY INFORMATION}

Structure of the membrane-intrinsic nitric oxide reductase from Roseobacter denitrificans

Allister Crow, Yuji Matsuda, Hiroyuki Arata, Arthur Oubrie

Supplemental Table 1: Crystallographic data and refinement statistics

Data Collection

Beam line

ESRF ID23-1

ESRF ID23-1

Wavelength $(\AA)$

0.9762

1.7385

Crystal Parameters

Space group

P $222_{1}$

P $22{ }_{1} 21$

Unit cell dimensions $(\AA)$

Unit cell angles $\left({ }^{\circ}\right)$
$a=86.2, b=98.8, c=126.8$
$a=85.3, b=98.7, c=125.6$
$90,90,90$
$90,90,90$

Dataset Statistics

Resolution range $(\AA)$

$53.36-2.85(3.00-2.85)$

51.30-3.60 (3.79-3.60)

Unique reflections

$25,236(3,124)$

$12,977(1,860)$

$R_{\text {sym }}$

$0.102(0.362)$

$0.112(0.373)$

$/ / \sigma(I)$

$13.3(3.4)$

$7.9(2.9)$

Completeness (\%)

$97.2(85.7)$

$98.8(99.5)$

Multiplicity

$6.7(4.7)$

$3.4(3.4)$

Refinement

Number of reflections

23,917

$R_{\text {work }}$

0.1885

$R_{\text {free }}$

0.2435

Rms bond lengths $(\AA)$

0.0091

Rms bond angles $\left({ }^{\circ}\right)$

1.5883

\section{Model statistics}

Ramachandran outliers

$1(0.2 \%)$

Number of Atoms (Protein)

4,726

Number of Atoms (Ligands/ions)

134

Number of Atoms (Waters) 15

B-factors (Protein) $\left(\AA^{2}\right)$

45

B-factors (Ligands) $\left(\AA^{2}\right)$

36

B-factors (Waters) $\left(\AA^{2}\right)$

40 


\begin{tabular}{|c|c|c|c|c|c|c|c|}
\hline & \multicolumn{2}{|c|}{$\lambda=1.7385 \AA$ Dataset } & \multicolumn{2}{|c|}{$\lambda=0.9762 \AA$ Dataset } & \multicolumn{2}{|c|}{ Ratio } & \multirow[t]{2}{*}{ Metal } \\
\hline & $\begin{array}{c}\text { Observe } \\
d\end{array}$ & $\begin{array}{c}\text { Theoretica } \\
\text { I }\end{array}$ & $\begin{array}{c}\text { Observe } \\
d\end{array}$ & $\begin{array}{c}\text { Theoretica } \\
\text { I }\end{array}$ & $\begin{array}{c}\text { Observe } \\
d\end{array}$ & $\begin{array}{c}\text { Theoretica } \\
l\end{array}$ & \\
\hline Heme $c$ & 4.1 & 3.9 & 1.5 & 1.5 & 0.4 & 0.4 & $\mathrm{Fe}$ \\
\hline Heme $b$ & 4.2 & 3.9 & 1.8 & 1.5 & 0.4 & 0.4 & $\mathrm{Fe}$ \\
\hline $\begin{array}{l}\text { Heme } b_{3} \\
\text { Active site }\end{array}$ & 3.4 & 3.9 & 1.2 & 1.5 & 0.3 & 0.4 & $\mathrm{Fe}$ \\
\hline metal & 4.0 & 3.9 & 1.5 & 1.5 & 0.4 & 0.4 & $\mathrm{Fe}$ \\
\hline $\begin{array}{l}\text { NorC metal } \\
\text { Bridging }\end{array}$ & 0.5 & 0.7 & 1.1 & 2.2 & 2.6 & 3.0 & $\mathrm{Cu}$ \\
\hline metal & 1.3 & 1.6 & 0.4 & 0.6 & 0.3 & 0.4 & $\mathrm{Ca}$ \\
\hline
\end{tabular}

Supplementary Table 2. Integrated anomalous scattering map values for each metal site in RdNOR. All values in electrons. Observed values correspond to the integrated peaks from the anomalous scattering maps. Theoretical f" values correspond to the assigned metal given in the rightmost column. 
$\alpha 1$

$\alpha 2$

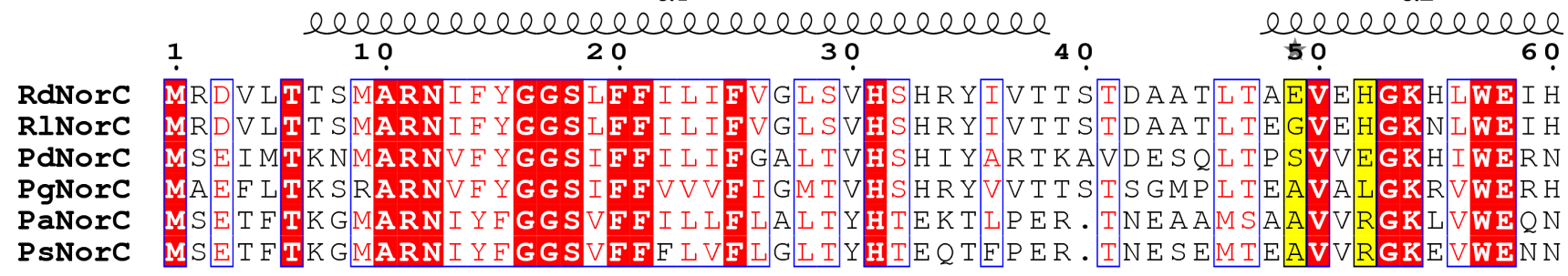

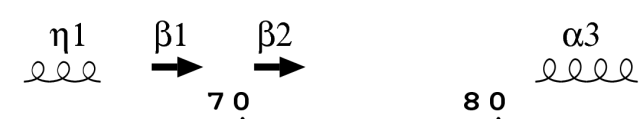

elee

$$
90 \text { lebelebelel }
$$

$\alpha 4$

110

120

RdNorC GCVNCHS I LEGAYFAPELGNVMTRWGVEDDPDAAFEALKGWMDAMPTG IEGRRQMPNFG

RINorC GCVNCHTILGEGAYFAP E L GNVM TRWGVEDDPEAAFE T LKGWMDAMP T G E GRR QMPNFG

PdNorC ACIDCHTLLGEGAYFAPELGNVMKRWGVQDDP D S A E TIKGWMESMP T G I E GRQMPRF

PgNorC SCINCHTLHGEGAYFAPEVGNVMTRWGVQDEPEEAFE I LN SWMES QP S GVEGRRQMPY FE

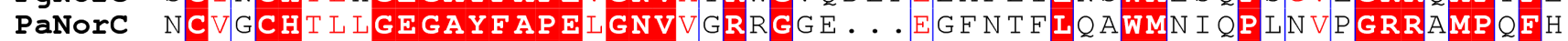

PsNorC NCIGCHSLLGEGAYFAPELGNVFVRRGGE ... ETFKP FLHAWMKA QP LA P GRRAMPQFN

$$
\begin{gathered}
\alpha 5 \\
\text { elebelebelele } \\
130
\end{gathered}
$$

RdNorC LNDEEYRATS DELLWTN TIR NQDWP PNDAC

RINorC IN D E E YRA L S D F L L WT T T I R N O D W P ND A

PdNorC ITDEEFRA L D F L L WT T T I T ONWP PNDAG

PgNorC ITE E E TRG L A E F L R W A D Q T D T Q G W P P ND A C

PaNorC IS E G QVDD L A E F L KW S S K I D T N Q W P P NKE G

PsNorC LS ElQQ QVD D M E E LKWT S K I D T NDW P P NK E G

Supplemental Figure 1. Multiple sequence alignment of the RdNOR small subunit and five key homologues in the region of the small subunit metal site. RdNorC - Roseobacter denitrificans; PaNorC - Pseudomonas aeruginosa; PdNorC-Paracoccus denitrificans; PsNorC-Pseudomonas stutzeri; RINorC-Roseobacter litoralis; PgNorC-Phaeobacter gallaeciensis. Secondary structure elements from the structure of RdNOR are shown immediately above the alignment. Locations of residues aligning with those coordinating the small subunit metal site in RdNOR are highlighted gold. 

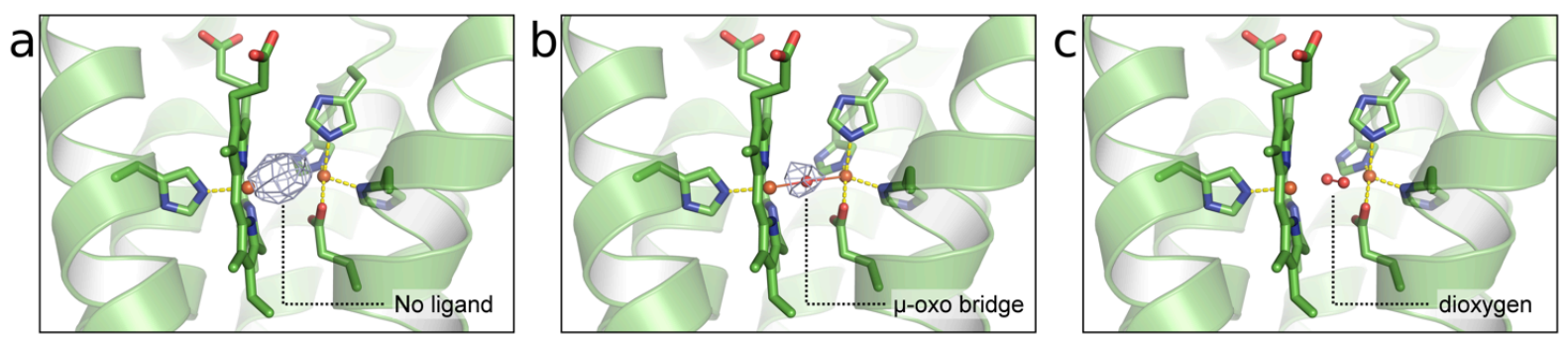

Supplemental Figure 2. Alternative models for the ligand bridging the active site site di-iron centre. The residual electron density for each model is shown as a blue mesh contoured at $5 \sigma$. (a) Active site without a bridging ligand. (b) A $\mu$-oxo bridge. (c) A bridging di-oxygen molecule. Iron atoms are shown as orange spheres. Only models with a diatomic ligand properly account for the observed electron density. While dioxygen is shown modelled in c, similar results were obtained for dinitrogen, carbon monoxide, nitric oxide, and a peroxo-bridge. 